\title{
Cooperative Transmission in Cloud RAN Considering Fronthaul Capacity and Cloud Processing Constraints
}

\author{
$\mathrm{Vu}$ Nguyen $\mathrm{Ha}^{\dagger}$, Long Bao Le ${ }^{\dagger}$, and Ngọc-Dũng Đào \\ ${ }^{\dagger}$ Institut National de la Recherche Scientifique-Énergie, Matériaux et Télécommunications (INRS-EMT), \\ Université du Québec, Montréal, Québec, Canada; e-mail: \{hanguyen,long.le\}@emt.inrs.ca. \\ ${ }^{\ddagger}$ Huawei Technologies Canada Co. Ltd., Ottawa, Canada; e-mail: daongocdung@ualberta.net.
}

\begin{abstract}
We investigate the cooperative transmission design for the cloud radio access network (C-RAN) considering fronthaul capacity and cloud processing constraints. Specifically, we consider the joint transmission scheme where the baseband signals and precoding vectors are processed and calculated by the cloud, which are delivered over the fronthaul links to the remote radio heads (RRHs) to form the $R F$ signals for being transmitted to the users. We formulate the joint optimization problem for precoding design and allocation of RRHs, fronthaul capacity, and $\mathrm{BBU}$ processing resources to minimize the total transmission power subject to QoS constraints of the users. We present both optimal exhaustive search algorithm and two lowcomplexity algorithms to solve the resource allocation problem where the first one can achieve the Pareto optimality and the second one can determine an efficient solution with pretty low complexity. Numerical results confirm the excellent performance of the proposed low-complexity algorithms.
\end{abstract}

Index Terms-Cloud radio access network (C-RAN), beamforming, power minimization, resource allocation.

\section{INTRODUCTION}

The next generation wireless cellular network is expected to provide significant enhancement in capacity to support emerging broadband applications. Coordinated multipoint (CoMP) transmission and reception techniques have been proposed as one of the most important solutions along this line for (LTE)-Advanced systems [1]. In a CoMP scheme, several base stations (BSs) cooperate their transmissions to mitigate the intra- and inter-cell interference. However, deployment of CoMP in cellular networks typically requires costly highspeed backhauls connecting different BSs for various CSI and information exchanges [2, 3]. C-RAN has been recently proposed as an alternative way to achieve the advantages of CoMP where we exploit the computation power of the cloud to compute and realize most base-band processing functionalities $[4,5]$. C-RAN can also enable us to deploy small-cells in existing wireless systems to improve the network coverage and capacity $[6,7]$.

In the C-RAN, main signal processing functions are performed by the digital baseband processing units (BBUs) in the cloud and the transmissions of radio signals to users are realized by remote radio heads (RRHs), which up-covert the received baseband signals from the cloud and transmit them in the RF frequency bands. There are various challenges in deploying the C-RAN including optimally utilizing the processing resource, efficiently using the fronthaul links connecting BBUs with distributed RRHs, and centralized control of the propagation signal. Some of these problems have been studied in recent works. In [8,9], the compression technique has been considered to reduce the amount of data transmitted over the backhaul links. [10] demonstrates the benefits of $\mathrm{C}$-RAN in the small-cell network. However, optimization of the joint transmission and allocation of the resources of $\mathrm{C}$ RAN (processing power and fronthaul capacity) has not been considered in the literature.

In this paper, we consider the design of joint transmission for transmission power minimization in C-RAN considering the constraints in the processing power and the fronthaul capacity as well as quality of services (QoS) for users. In particular, we investigate how to optimize the set of RRHs serving each user as well as their associated precoding vectors subject to all system and QoS constraints. Toward this end, we present an optimal resource allocation problem, which turns out to be an NP-hard non-linear mixed integer program. We then describe an optimal exhaustive search method to determine the optimal solution. In addition, we propose two lowcomplexity algorithms to obtain efficient solutions. Finally, numerical results are presented to illustrate the performance of the proposed algorithms.

The remaining of this paper is organized as follows. We describe the system model and problem formulation in Section II. In Section III, we present the feasibility and an optimal exhaustive search algorithm. Low-complexity algorithms are described in section IV. Numerical results are presented in Section V followed by conclusion in Section VI. For notation, we use $\mathbf{X}^{T}, \mathbf{X}^{H}, \operatorname{Tr}(\mathbf{X})$ and $\operatorname{rank}(\mathbf{X})$ to denote the normal transpose, Hermitian transpose, trace and rank of matrix $\mathbf{X}$, respectively. $\mathbf{1}_{x \times y}, \mathbf{0}_{x \times y}$ and $\mathbf{I}_{x \times y}$ denote the matrix of ones, matrix of zeros, and an identify matrix whose dimension are $x \times y$, respectively.

\section{System Model AND Problem Formulation}

\section{A. Overview of $C-R A N$}

The general architecture of C-RAN is illustrated in Fig. 1, which consist of three main components, namely (i) central- 


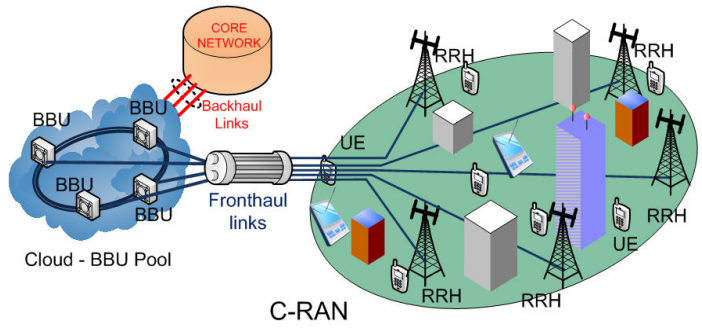

Fig. 1. The Cloud-RAN architecture.

ized processors or BBUs pool, (ii) the optical transport network (i.e., fronthaul links), and (iii) remote radio head (RRH) access units with antennas located at the remote sites. The processing center comprising a large number of BBUs is the heart of this architecture where BBUs operate as virtual base stations to process baseband signals for users and optimize the network resource allocation tasks for the system.

The transport network connects the central processing pool and the distributed RRH units. Optical fiber using wavelength division multiple access (WDM) and/or time division multiplexing (TDM) are typically employed to carry baseband signals from the BBUs to the RRHs. The RRHs transmit the $\mathrm{RF}$ signals formed by using baseband signals received from BBUs to user equipments (UEs) in the downlink or forward the baseband signals from UEs to the BBU pool for further processing in the uplink. By conducting most signal processing functions in the cloud (i.e., by BBU pool), RRHs can be relatively simple, which can be distributed in the large network in a cost-efficient manner.

\section{B. System Model and Transmission Strategy}

We consider the C-RAN where there are $B$ BBUs in the cloud connecting with $K$ RRHs to support downlink communications for $M$ UEs. Each BBU processes baseband signals for a separate set of UEs and RRHs up-convert these baseband signals to the RF band to transmit to the UEs. Denote $\mathcal{U}^{b}$ as the set of UEs whose baseband signals are processed by BBU $b(b=1, \ldots, B)$. Let $\mathbf{A} \in \mathbb{R}^{B \times M}$ be the matrix representing the matching among BBUs and UEs where

$$
\mathbf{A}(b, u)=a_{u}^{b}= \begin{cases}1 & \text { if } \mathrm{UE} u \text { is supported by BBU } b \\ 0 & \text { otherwise. }\end{cases}
$$

We also define the sets $\mathcal{U}^{b}=\left\{u \mid 1 \leq u \leq M, a_{u}^{b}=1\right\}$. Due to the limited processing capacity of BBUs [4], we assume that each BBU can only support at most $o^{b}$ UEs. In addition, each UE is assumed to be served by only one BBU in the BBU pool. Then, we have the following constraints.

$$
\sum_{u=1}^{M} a_{u}^{b} \leq o^{b}, b=1, \ldots, B, \text { and } \sum_{b=1}^{B} a_{u}^{b}=1, u=1, \ldots, M .
$$

We assume that each UE receives signals from a specific group of RRHs, which process the data from the corresponding supporting BBU. This joint transmission scheme aims to exploit the degree of freedoms available at different RRHs to mitigate the interference [1], [2]. This helps improve the performance of the whole network, i.e., increasing the throughput and/or reducing the transmission power. Let $\mathbf{T} \in \mathbb{R}^{K \times M}$ be the matrix that represents the matching of serving RRHs for UEs where

$$
\mathbf{T}(k, u)=t_{u}^{k}= \begin{cases}1 & \text { if RRH } k \text { forwards signal to UE } u \\ 0 & \text { otherwise. }\end{cases}
$$

It can be observed that the fronthaul link between RRH $k$ and BBU $b$ will carry the baseband signal for UE $u$ if and only if $a_{u}^{b} t_{u}^{k}=1$. Denote $\mathcal{R}_{u}$ as the set of RRHs who serve UE $u$. This set can be determined as

$$
\mathcal{R}_{u}=\left\{k \mid k \in \mathcal{R}, t_{u}^{k}=1\right\},
$$

where $\mathcal{R}$ represents the set of all RRHs. To model the limited capacity of the fronthaul links in C-RAN, we assume that the fronthaul link between BBU $b$ and RRH $k$ can carry at most $c_{k}^{b}$ baseband signals. Then, we have another set of constraints capturing this limited fronthaul capacity as

$$
\sum_{u=1}^{M} a_{u}^{b} t_{u}^{k} \leq c_{k}^{b}, \quad k=1, \ldots, K ; b=1, \ldots, B .
$$

The transmission strategy in C-RAN can be summarized as follows. After allocating the BBUs and RRHs to serve UEs, the BBU $b$ collects the data of UE $u$ in set $\mathcal{U}^{b}$ from the core network, process baseband signals for these UEs, and determine the precoding vectors for the corresponding RRHs. Then, it sends the baseband signals and precoding vectors of UE $u$ to the serving RRHs in the set $\mathcal{R}_{u}$ over the fronthaul link. Finally, each RRH will up-convert the received signals to the RF band and transmit to UEs using the received precoding vectors.

Remark 1. We have not considered thefeedback of channel state information (CSI) from RRH to BBU pool for processing the baseband signals. In practice, a small fraction of the fronthaul capacity can be allocated for this CSI feedback, whose optimization is outside of scope of the current work.

\section{Problem Formulation}

In this paper, we consider the MISO transmission from RRHs to UEs where RRH $k$ is equipped with $N_{k}$ antennas and each UE has a single antenna. We assume that UE $u$ will receive a symbol sequence $x_{u} \in \mathbb{C}$ of unit power, which is transmitted from RRHs in set $\mathcal{R}_{u}$ upon receiving the processed baseband signals from BBU $b_{u}$. For BBU and RRH allocation solutions represented by $\mathbf{A}$ and $\mathbf{T}$, the corresponding baseband signal $y_{u}$ received at UE $u$ can be expressed as

$$
y_{u}=\underbrace{\sum_{k \in \mathcal{R}_{u}} \mathbf{h}_{u}^{k H} \mathbf{v}_{u}^{k} x_{u}}_{\text {desired signal }}+\underbrace{\sum_{i=1, \neq u}^{M} \sum_{l \in \mathcal{R}_{i}} \mathbf{h}_{u}^{l H} \mathbf{v}_{i}^{l} x_{i}}_{\text {interference }}+z_{u},
$$

where $\mathbf{h}_{u}^{k} \in \mathbb{C}^{N_{k} \times 1}$ denote the channel coefficients between RRH $k$ and UE $u, \mathbf{v}_{u}^{k} \in \mathbb{C}^{N_{l} \times 1}$ is the precoding vector at RRH $k$ corresponding to the signal transmitted to $\mathrm{UE} u$, and $z_{u}$ 
describes the noise at UE $u$. Then, the signal-to-interferenceplus-noise-ratio (SINR) achieved by UE $u$ for a given matrix $\mathbf{T}$ can be written as

$$
\Gamma_{u}(\mathbf{T}, \mathbf{V})=\frac{\left|\sum_{k \in \mathcal{R}_{u}} \mathbf{h}_{u}^{k H} \mathbf{v}_{u}^{k}\right|^{2}}{\sum_{i=1, \neq u}^{M}\left|\sum_{l \in \mathcal{R}_{i}} \mathbf{h}_{u}^{l H} \mathbf{v}_{i}^{l}\right|^{2}+\sigma^{2}} .
$$

where $\mathbf{V}=\left[\mathbf{v}_{1}^{1}, \ldots, \mathbf{v}_{M}^{1}, \mathbf{v}_{1}^{2}, \ldots, \mathbf{v}_{M}^{2}, \cdots, \mathbf{v}_{1}^{K}, \ldots, \mathbf{v}_{M}^{K}\right]$ and $\sigma^{2}$ is the noise power. We assume that each UE $u$ in the network aims to maintain its required quality-of-service (QoS) which is represented by the corresponding target SINR $\bar{\gamma}_{u}$. This QoS requirement can be described by the following constraints

$$
\Gamma_{u}(\mathbf{T}, \mathbf{V}) \geq \bar{\gamma}_{u}, \quad u=1, \ldots, M .
$$

We are interested in determining the matching of BBUs, RRHs with UEs (i.e., represented by matrices $\mathbf{A}$ and $\mathbf{T}$, respectively) and the precoding vectors for all RRHs to minimize the total transmission power while supporting the QoS for all UEs. This design can be formulated into the following optimization problem

$$
\begin{aligned}
& \min _{\mathbf{A}, \mathbf{T}, \mathbf{V}} \sum_{k=1}^{K} \sum_{u=1}^{M} t_{u}^{k} \mathbf{v}_{u}^{k H} \mathbf{v}_{u}^{k} \\
& \text { s.t. } \quad \text { constraints }(2),(5),(8) .
\end{aligned}
$$

The optimization problem (9) is indeed a non-linear mixed integer quadratic program, which is an NP-hard problem. In the following, we propose an optimal exhaustive search method as well as low-complexity algorithms to solve this problem.

\section{OPTIMAL SOLUTION}

\section{A. Precoding Design and BBU Allocation Given $\mathbf{T}$}

1) Precoding Design: To gain insight into the optimal structure of the optimization problem (9), we investigate the precoding design for the given RRH allocation (i.e., matrix T). We first discuss how to capture the constraint imposed by the chosen $\mathbf{T}$ in the precoding design problem. To represent the precoding solution for any $\mathbf{T}$, let $\mathbf{v}_{u} \in \mathbb{C}^{N \times 1}$ ( $\left.N=\sum_{k=1}^{K} N_{k}\right)$ denote the precoding over all RRHs, which is defined as $\mathbf{v}_{u}=\left[\mathbf{v}_{u}^{1 H} \mathbf{v}_{u}^{2 H} \ldots \mathbf{v}_{u}^{K H}\right]^{H}$ where $\mathbf{v}_{u}^{k H}=$ $\mathbf{0}_{\left(N_{k} \times 1\right)}$ if $t_{u}^{k}=0$. This basically means that the precoding vector is equal to zero for any RRH that does not serve the underlying UE.

Let us define $\mathbf{e}_{u}=\mathbf{v}_{u}-\mathbf{D}_{u} \mathbf{v}_{u}$ and $\mathbf{D}_{u}=$ $\operatorname{diag}\left(\left[t_{u}^{1} \times \mathbf{1}_{\left(1 \times N_{1}\right)} \ldots t_{u}^{K} \times \mathbf{1}_{\left(1 \times N_{K}\right)}\right]^{T}\right)$ then we need to impose the constraints $\mathbf{e}_{u}^{H} \mathbf{e}_{u}=0$ so that only serving RRHs set non-zero precoding vectors for the underlying UE. Now, we define $\mathbf{W}_{u}=\mathbf{v}_{u} \mathbf{v}_{u}^{H}$ where $\mathbf{W}_{u} \in \mathbb{C}^{N \times N}$. Using the fact that $\mathbf{x}_{u}^{H} \mathbf{x}_{u}=\operatorname{Tr}\left(\mathbf{x}_{u} \mathbf{x}_{u}^{H}\right)$ and the definition of $\mathbf{W}_{u}$, the $\mathbf{e}_{u}^{H} \mathbf{e}_{u}=0$ is then equivalent to

$$
\operatorname{Tr}\left[\left(\mathbf{I}_{(N \times N)}-\mathbf{D}_{u}\right) \mathbf{W}_{u}\right]=0 .
$$

Moreover, $\mathbf{W}_{u}$ is positive semi-definite $\left(\mathbf{W}_{u} \succeq \mathbf{0}\right)$ and has rank one because it is generated from vector $\mathbf{v}_{u}$ (recall that
$\mathbf{W}_{u}=\mathbf{v}_{u} \mathbf{v}_{u}^{H}$ ). Instead of finding vector $\mathbf{v}_{u}$, we can first determine matrix $\mathbf{W}_{u}$, which is positive semi-definite, has rank one, and satisfies (10). Similarly, we define the channel vector $\mathbf{h}_{u}=\left[\mathbf{h}_{u}^{1 H} \mathbf{h}_{u}^{2 H} \ldots \mathbf{h}_{u}^{K H}\right]^{H}$ and $\mathbf{H}_{u}=\mathbf{h}_{u} \mathbf{h}_{u}^{H}$ where we have $\mathbf{h}_{u} \in \mathbb{C}^{N \times 1}$ and $\mathbf{H}_{u} \in \mathbb{C}^{N \times N}$. Then, it can be verified that the SINR constraint for UE $u$ in (8) can be rewritten in the matrix form as

$$
\operatorname{Tr}\left(\mathbf{H}_{u} \mathbf{W}_{u}\right)-\bar{\gamma}_{u} \sum_{i=1, \neq u}^{M} \operatorname{Tr}\left(\mathbf{H}_{u} \mathbf{W}_{i}\right) \geq \bar{\gamma}_{u} \sigma^{2} .
$$

Hence, for a given matrix $\mathbf{T}$, the precoding design can be formulated as the following optimization problem

$$
\begin{aligned}
\underset{\left\{\mathbf{W}_{u}\right\}_{u=1}^{M}}{\min } & \sum_{u=1}^{M} \operatorname{Tr}\left(\mathbf{W}_{u}\right) \\
\text { s.t. } & \text { constraints }(10),(11) \\
& \mathbf{W}_{u} \succeq \mathbf{0}, \operatorname{rank}\left(\mathbf{W}_{u}\right)=1, \forall u .
\end{aligned}
$$

This transformation reveals structure of the precoding design problem. Specifically, if we remove the rank-one constraints $\operatorname{rank}\left(\mathbf{W}_{u}\right)=1$ from (12) then the resulting problem is convex. In fact, this relaxed problem is the semi-definite program (SDP), which is convex and therefore can be solved easily by using standard tools such as CVX solver [11]. If the obtained solution $\mathbf{W}_{u}^{*}(\mathbf{T})$ from this relaxed problem has rank larger than one, we can use the eigenvector approximation technique [12] to find $\mathbf{v}_{u}^{*}(\mathbf{T})$. In particular, $\mathbf{v}_{u}^{*}(\mathbf{T})$ will be approximated as the eigenvector corresponding to the largest modulus eigenvalue.

2) BBU Allocation: For a given matrix $\mathbf{T}$ and precoding design, we still need to determine the BBU allocation (i.e., to find matrix A) which satisfies the constraints (2), and (5). Toward this end, let us define the following vectors $\mathbf{t}_{k}=$ $\left[t_{1}^{k} t_{2}^{k} \ldots t_{M}^{k}\right]^{T}, \mathbf{o}=\left[o^{1} o^{2} \ldots o^{B}\right]^{T}$ and $\mathbf{c}_{k}=\left[c_{1}^{k} c_{2}^{k} \ldots c_{B}^{k}\right]^{T}$, $\mathbf{a}=\left[a_{1}^{1} \ldots a_{M}^{1} a_{1}^{2} \ldots a_{M}^{2} \ldots a_{1}^{B} \ldots a_{M}^{B}\right]^{T}$. Then, it can be verified that matrix $\mathbf{A}$ can be determined by solving the following binary integer program

$$
\begin{aligned}
& \min _{\mathbf{a}} \mathbf{1}_{(1 \times B M)} \mathbf{a} \\
& \text { s.t. } \mathbf{F} \cdot \mathbf{a} \leq \mathbf{c}, \\
& \mathbf{E} \cdot \mathbf{a}=\mathbf{1}_{M \times 1},
\end{aligned}
$$

where $\mathbf{F} \in \mathbb{R}^{(B+B K) \times B M}, \mathbf{E} \in \mathbb{R}^{M \times B M}$ and $\mathbf{c}$ are defined as $\mathbf{c}=\left[\begin{array}{llll}\mathbf{o}^{T} & \mathbf{c}_{1}^{T} & \cdots & \mathbf{c}_{K}^{T}\end{array}\right]^{T}, \mathbf{E}=\left[\begin{array}{llll}\mathbf{I}_{M \times M} & \mathbf{I}_{M \times M} & \cdots & \mathbf{I}_{M \times M}\end{array}\right]$ and

$$
\mathbf{F}=\left[\begin{array}{cccccccccc}
\mathbf{1}_{(M \times 1)} & \ldots & \mathbf{0} & \mathbf{t}_{1} & \ldots & \mathbf{0} & & \mathbf{t}_{K} & \ldots & \mathbf{0} \\
\vdots & \ddots & \vdots & \vdots & \ddots & \vdots & \ldots & \vdots & \ddots & \vdots \\
\mathbf{0} & \ldots & \mathbf{1}_{(M \times 1)} & \mathbf{0} & \ldots & \mathbf{t}_{1} & & \mathbf{0} & \ldots & \mathbf{t}_{K}
\end{array}\right]^{T}
$$

This problem can be solved by using the binary integer programming algorithm [13] or solver such as function "bintprog” in MATLAB.

\section{B. Optimal Exhaustive Search Method}

Note that it is not always possible to find the vector $\mathbf{V}$ and BBU allocation solution $\mathbf{A}$ from the above optimization 
problems (12) and (13) for a given $\mathbf{T}$. This motivates us to formalize the feasibility of $\mathbf{T}$ in the following definition.

Definition 1. A matrix $\mathbf{T}$ is called feasible if and only if it is possible to determine $\mathbf{V}$ and $\mathbf{A}$ from the optimization problems (12) and (13) for the given $\mathbf{T}$, respectively.

We are now ready to describe the optimal exhaustive search method. We first generate all possible $2^{K M} \mathrm{RRH}$ allocation solutions T. Then, for each specific RRH allocation solution, we obtain the precoding vectors and BBU allocation solutions by solving (12) and (13) if it is feasible to do so. Then, the optimal solution of our problem (9) is the one that achieves the minimum power in (9) among feasible RRH allocation solutions $\mathbf{T}$ in the set of all possible $2^{K M}$ matrices $\mathbf{T}$. It is evident that this exhaustive search method has exponential complexity, which would be prohibitive if the number of RRHs and UEs are very large.

\section{LOW-COMPLEXITY ALgORITHMS}

We will present two low-complexity algorithms in this section.

\section{A. Motivation}

Denote $\mathcal{Z}(\mathbf{T})=\left\{(k, u) \mid t_{u}^{k}=0\right\}$ as the set of all zero elements of $\mathbf{T}$. In the following, we introduce some new notations and concepts that help quantify the quality of a feasible solution.

Definition 2. RRH allocation matrix $\mathbf{T}$ is called bigger than $\mathbf{T}^{\prime}, \mathbf{T} \triangleright \mathbf{T}^{\prime}$ if and only if

$$
\mathcal{Z}(\mathbf{T}) \subset \mathcal{Z}\left(\mathbf{T}^{\prime}\right) .
$$

Definition 3. RRH allocation matrix $\mathbf{T}^{\dagger}$ is called Pareto optimal if it is feasible and there exist no feasible $\mathbf{T}$ such that $\mathbf{T} \triangleright \mathbf{T}^{\dagger}$.

Let $\pi(\mathbf{T})$ be the objective value of (12) for the RRH allocation matrix $\mathbf{T}$. In the following, we say $\mathbf{T}$ is feasible (infeasible) for $\mathbf{V}$ or for $\mathbf{A}$ if there exist (not exist) a feasible solution for problems (12) and (13), respectively. We have following results.

\section{Lemma 1. If $\mathbf{T}$ is bigger than $\mathbf{T}^{\prime}$ then we have}

1) If $\mathbf{T}$ is infeasible for $\mathbf{V}$ then $\mathbf{T}^{\prime}$ is infeasible for $\mathbf{V}$.

2) If $\mathbf{T}^{\prime}$ is infeasible for $\mathbf{A}$ then $\mathbf{T}$ is infeasible for $\mathbf{A}$.

3) If $\mathbf{T}^{\prime}$ and $\mathbf{T}$ are both feasible for $\mathbf{V}$ then $\pi(\mathbf{T}) \leq \pi\left(\mathbf{T}^{\prime}\right)$.

Proof. The lemma can be proved by studying the feasible sets of problems (12) and (13) for each case. Let $\Omega(\mathbf{T})$ and $\Phi(\mathbf{T})$ be the feasible sets of problems (12) and (13) for the given matrix $\mathbf{T}$, respectively. For $\mathcal{Z}(\mathbf{T}) \subset \mathcal{Z}\left(\mathbf{T}^{\prime}\right)$, it can be verified that $\Omega\left(\mathbf{T}^{\prime}\right) \subseteq \Omega(\mathbf{T})$ and $\Phi(\mathbf{T}) \subseteq \Phi\left(\mathbf{T}^{\prime}\right)$. Therefore, the first two statements of the lemma are proved. The proof for the last statement can be completed similarly.

This lemma implies that the bigger $\mathbf{T}$ results in the better performance. This motivates us to develop an algorithm that finds an efficient $\mathbf{T}$ so that each UE is served by as many RRHs as possible.

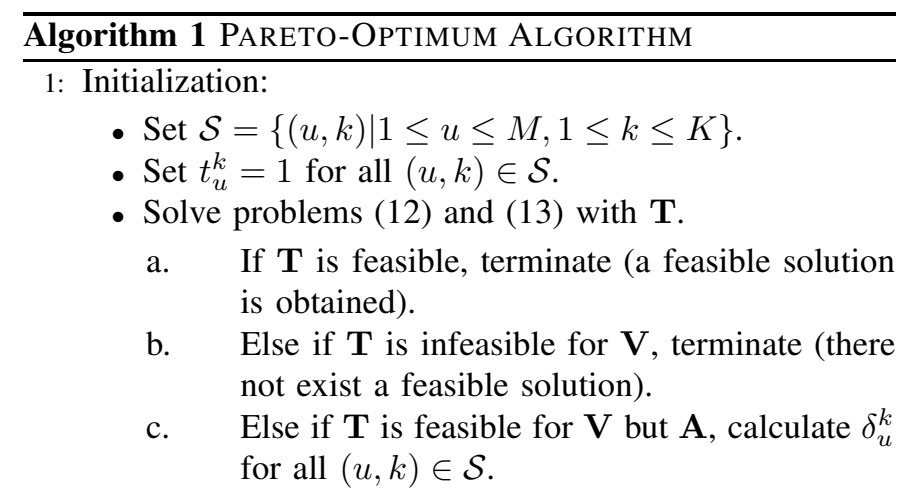

2: If $\mathcal{S}=\varnothing$, terminate (no feasible solution is obtained). Else, set $t_{u^{\prime}}^{k^{\prime}}=0$ for $\left(u^{\prime}, k^{\prime}\right)=\operatorname{argmin}_{(u, k) \in \mathcal{S}} \delta_{u}^{k}$ and remove this link from $\mathcal{S}$, i.e., update $\mathcal{S}=\mathcal{S} /\left(u^{\prime}, k^{\prime}\right)$.

3: Solve problems (12) and (13) with current $\mathbf{T}$.

a. If $\mathbf{T}$ is feasible, update $\mathcal{S}_{p}=\mathcal{Z}(\mathbf{T})$, go to Step 4.

b. Else if $\mathbf{T}$ is infeasible for $\mathbf{V}$, set $t_{u^{\prime}}^{k^{\prime}}=1$ and go to Step 2.

c. $\quad$ Else if $\mathbf{T}$ is feasible for $\mathbf{V}$ but $\mathbf{A}$, update $\delta_{u}^{k}$ for all $(u, k) \in \mathcal{S}$ and go to Step 2.

4: If $\mathcal{S}_{p}=\varnothing$, terminate. Else, choose each $\left(u^{\prime \prime}, k^{\prime \prime}\right) \in \mathcal{S}_{p}$, set $t_{u^{\prime \prime}}^{k^{\prime \prime}}=1$ and $\mathcal{S}_{p}=\mathcal{S}_{p} /\left(u^{\prime \prime}, k^{\prime \prime}\right)$.

a. If $\mathbf{T}$ is feasible for $\mathbf{A}$, update $\mathbf{T}$.

b. Else if $\mathbf{T}$ is infeasible for $\mathbf{A}$, set $t_{u^{\prime \prime}}^{k^{\prime \prime}}=0$ and go to Step 4.

\section{B. Pareto-optimum Algorithm}

The key idea behind this algorithm is that we start with largest possible $\mathbf{T}$ where each UE is served by all RRHs. If this RRH allocation is infeasible for $\mathbf{V}$ and/or $\mathbf{A}$, we greedily drop one weak link due to the current $\mathbf{T}$ in each step to obtain a feasible solution. For a given matrix $\mathbf{T}$, which is feasible for $\mathbf{V}$, let $\mathbf{v}_{u}^{k *}(\mathbf{T})$ be the optimal precoding vector at RRH $k$ by solving problem (12). Then, we propose the following metric that quantifies that the relative contribution of a link from RRH $k$ for UE $u$ to SINR achieved by UE $u$ as follows:

$$
\delta_{u}^{k}=\frac{\left|\mathbf{h}_{u}^{k H} \mathbf{v}_{u}^{k *}\right|^{2}}{\left|\sum_{l \in \mathcal{R}} \mathbf{h}_{u}^{l H} \mathbf{v}_{u}^{l *}\right|^{2}} .
$$

The low-complexity Pareto-optimum algorithm is presented in Algorithm 1. Initially, we set $\mathbf{T}=\mathbf{1}_{(K \times M)}$. If it is feasible to find both $\mathbf{V}$ and $\mathbf{A}$, we obtain the optimal solution. If $\mathbf{T}$ is infeasible for finding $\mathbf{V}$, there is no feasible solution for our problem (9). This can be concluded by the result in the first statement of Lemma 1. Otherwise, if $\mathbf{T}$ is feasible for finding $\mathbf{V}$ but $\mathbf{A}$, we seek to drop some weak links from RRHs to UEs to obtain a feasible solution. Dropping a particular link is achieved by turning the corresponding " 1 " element of $\mathbf{T}$ to zero (in Step 2 and Step 3 of Algorithm 1).

In this algorithm, $\mathcal{S}$ presents the set of links (i.e., elements of $\mathbf{T}$ ) that have not been considered for being dropped yet. In each iteration, we solve problems (12) and (13) again after updating $\mathbf{T}$. The algorithm terminates in Step 1.a and 1.b since we can conclude that the (9) is feasible or infeasible with the 
initial RRH allocation $\mathbf{T}$, respectively. If $\mathbf{T}$ is feasible for $\mathbf{V}$ but $\mathbf{A}$ in Step 1.c, we proceed to drop one weakest link with minimum metric given in (15) in Step 2. If the resulting $\mathbf{T}$ is feasible, we update $\mathcal{S}_{p}=\mathcal{Z}(\mathbf{T})$, which represent the set of off links; then we go to Step 4. In Step 4, we attempt to turn on one link $\left(u^{\prime \prime}, k^{\prime \prime}\right) \in \mathcal{S}_{p}$ that has been turned off in the previous step. This is required to achieve the Pareto-optimum solution as presented in the following Lemma.

In Step 3.b, if $\mathbf{T}$ is infeasible for $\mathbf{V}$, we turn on back the link since it does not help resolve the infeasibility. Otherwise, if $\mathbf{T}$ is feasible for $\mathbf{V}$ but $\mathbf{A}$, we will go back Step 2 and attempt to turn off more link. The algorithm terminates finally as a feasible solution is obtained or there is no link remaining in the set $\mathcal{S}$. After step 2 or step 4 of each iteration, the number of elements in sets $\mathcal{S}$ and $\mathcal{S}_{p}$ decreases by one, respectively. It can be verified that the algorithm will terminate in at most $2 K M$ iterations. Therefore, our proposed algorithm has much lower complexity in comparison with the optimal exhaustive search method. We show that the obtained solution by Algorithm 1 is Pareto optimum in the following lemma.

Lemma 2. The feasible solution $\mathbf{T}^{*}$ returned by Algorithm 1 (if exist) is Pareto optimum.

Proof. A feasible solution $\mathbf{T}^{*}$ is obtained if $\mathbf{T}^{*}=\mathbf{1}_{(K \times M)}$ or $\mathbf{T}^{*}$ in step $3 \mathrm{a}$ is feasible. If $\mathbf{T}^{*}=\mathbf{1}_{(K \times M)}$, it is obviously a Pareto optimum solution. If $\mathbf{T}^{*} \neq \mathbf{1}_{(K \times M)}$, let $\mathbf{T}^{(0)}$ be the feasible $\mathbf{T}$ obtained in step 3a before we proceed to Step 4 . Let $\mathbf{T}^{(l)}$ be the feasible $\mathbf{T}$ obtained after performing the $l^{\text {th }}$ sub-iteration in Step 4 and $(k, u)^{(l)}$ be element processed in that sub-iteration where $l=1, \ldots, L$ and $L$ is the number of elements in set $\mathcal{Z}\left(\mathbf{T}^{(0)}\right)$. We have $\mathbf{T}^{(L)}=\mathbf{T}^{*}$ and $\mathcal{Z}\left(\mathbf{T}^{(n)}\right) \subseteq$ $\mathcal{Z}\left(\mathbf{T}^{(l)}\right)$ if $n>l$.

If $\mathbf{T}^{*}$ is not a Pareto optimum solution, there will be a feasible $\mathbf{T}^{\dagger}$ which is bigger than $\mathbf{T}^{*}\left(\mathbf{T}^{\dagger} \triangleright \mathbf{T}^{*}\right)$. We will prove Lemma 2 by showing that this IF results in a contradiction. Since $\mathcal{Z}\left(\mathbf{T}^{\dagger}\right) \subset \mathcal{Z}\left(\mathbf{T}^{*}\right)$, an element $\left(k^{\prime}, u^{\prime}\right)$ which satisfies $\left(k^{\prime}, u^{\prime}\right) \in \mathcal{Z}\left(\mathbf{T}^{*}\right)$ and $\left(k^{\prime}, u^{\prime}\right) \notin \mathcal{Z}\left(\mathbf{T}^{\dagger}\right)$ can be determined. Then, $\left(k^{\prime}, u^{\prime}\right)$ must be a member in the sets $\mathcal{Z}\left(\mathbf{T}^{(l)}\right)(l=$ $0, \ldots, L)$ and it must have been processed in one sub-iteration of Step 4. Assume $\left(k^{\prime}, u^{\prime}\right)=(k, u)^{\left(l^{\prime}\right)}$. According to Step 4 of Algorithm 2, we have $\mathbf{T}^{\prime}$ where $\mathcal{Z}\left(\mathbf{T}^{\prime}\right)=\mathcal{Z}\left(\mathbf{T}^{\left(l^{\prime}\right)}\right) /\left(k^{\prime}, u^{\prime}\right)$ is not feasible. However, $\mathbf{T}^{\prime}$ is feasible for $\mathbf{A}$ because $\mathcal{Z}\left(\mathbf{T}^{\dagger}\right) \subset$ $\mathcal{Z}\left(\mathbf{T}^{\left(l^{\prime}\right)}\right)$ and $\left(k^{\prime}, u^{\prime}\right) \notin \mathcal{Z}\left(\mathbf{T}^{\dagger}\right)$ result in $\mathcal{Z}\left(\mathbf{T}^{\dagger}\right) \subset \mathcal{Z}\left(\mathbf{T}^{\prime}\right)$ (the statement 2 of Lemma 1). Moreover, $\mathbf{T}^{\prime}$ is also feasible for $\mathbf{V}$ because $\mathcal{Z}\left(\mathbf{T}^{\prime}\right) \subset \mathcal{Z}\left(\mathbf{T}^{\left(l^{\prime}\right)}\right)$; hence, $\mathbf{T}^{\prime}$ is feasible. This results in a contradiction; therefore, $\mathbf{T}^{*}$ must be a Pareto optimum solution.

\section{Fast Algorithm}

We propose an even faster algorithm in this section where we determine a potential BBUs allocation first then start dropping links connecting RRHs with UEs to meet the capacity constraints of fronthaul links between BBUs and RRHs. Let $\mu_{u}^{k}$ describe the weight of the link from RRH $k$ to UE $u$ representing the quality of this connection. For example, this weight can be the proposed metric in (15) or the average channel gain $\left(L_{u}^{k}\right)$ of this link. In the numerical studies, we

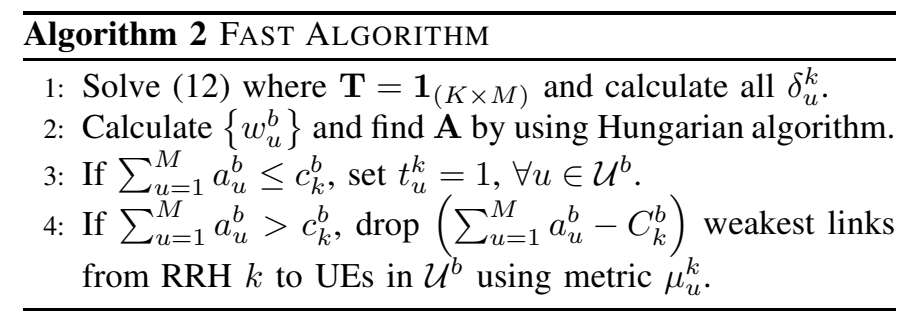

employ the metric in (15) as the assignment weight. Then, we express the weight corresponding the case where BBU $b$ processes the baseband signal for UE $u$ as

$$
w_{u}^{b}=\sum_{k \in \mathcal{R}} w_{k, u}^{b}=\sum_{k \in \mathcal{R}} c_{k}^{b} \mu_{u}^{k}
$$

Then, the matching between BBUs and UEs can be determined to maximize the total weights, i.e., we need to solve the following optimization problem

$$
\begin{aligned}
& \max _{\mathbf{A}} \sum_{b=1}^{B} \sum_{u=1}^{M} a_{u}^{b} w_{u}^{b} \\
& \text { s.t. constraint (2). }
\end{aligned}
$$

This optimization problem can be transformed into the standard matching problem as follows. Suppose we create $o^{b}$ virtual BBUs $\left(\left\{b_{1}, \ldots, b_{o^{b}}\right\}\right)$ for each BBU $b$ where the weight $w_{u}^{b_{n}}\left(n=1, \ldots, o^{b}\right)$ representing the matching between BBU $b_{n}$ and UE $u$ is equal to $w_{u}^{b}$. We can view the problem (17) as the one-to-one matching problem between $\sum_{b=1}^{B} o^{b}$ virtual BBUs and $M$ UEs. The solution of this transformed matching problem can be obtained by using the well-known Hungarian algorithm (Algorithm 14.2.3 given in [14]). After running the Hungarian algorithm, if there is a virtual BBU $b_{n}, n \in\left\{1, \ldots, o^{b}\right\}$ which is matched with UE $u$ then UE $u$ will be served by BBU $b$, i.e., $a_{u}^{b}=1$; otherwise, we set $a_{u}^{b}=0$. Based on the achieved $\mathbf{A}$, we may need to turn off some weak links to meet the fronthaul capacity constraints, which is described in Algorithm 2.

\section{Numerical RESUlts}

The small network is employed to evaluate the efficacy of the low-complexity algorithms where UEs are randomly located inside a square and the four RRHs are in the centers of these squares whose edge length is $50 \mathrm{~m}$. The channel gains are generated by considering both Rayleigh fading and path loss which is modeled as $L_{u}^{k}=36.8 \log _{10}\left(d_{u}^{k}\right)+43.8+$ $20 \log _{10}\left(\frac{f_{c}}{5}\right)$, where $d_{u}^{k}$ is the distance from UE $k$ to RRH $k$; $f_{c}=2.5 \mathrm{GHz}$. The noise power is set equal $\sigma^{2}=10^{-13} \mathrm{~W}$. The number of BBUs is 3 and number of antennas at each RRH is 3 . The values of $o_{b}$ and $c_{k}^{b}$ are randomly chosen in sets $\left\{1, \ldots, O_{\max }\right\}$ and $\left\{1, \ldots, C_{\max }\right\}$, respectively.

In Fig. 2, we show the total transmission power of all RRHs versus the target SINR of UEs $\bar{\gamma}_{u}$ for all three algorithms. The number of UEs in this simulation is 6 and the largest number of links from RRH to BBU can be supported is 5. As $\bar{\gamma}_{u}$ increases, the required power and interference also increase, which results in the fast increase in the total transmission power. In addition, this figure confirms that our proposed 


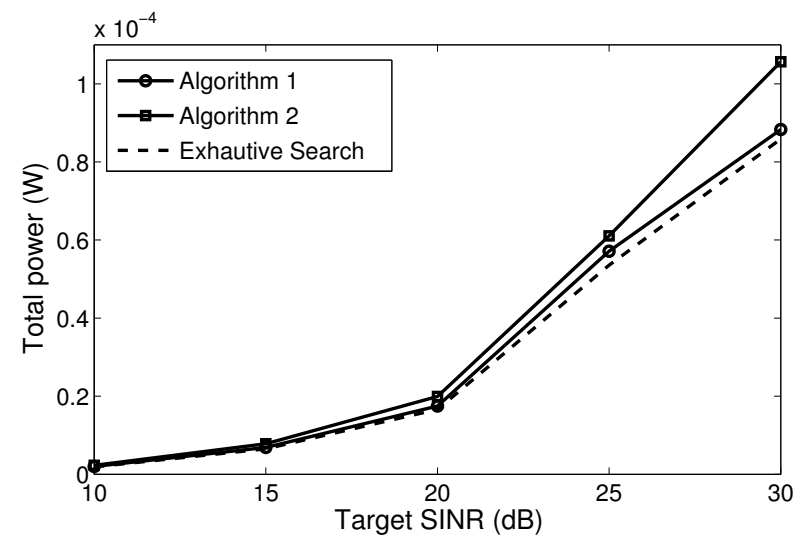

Fig. 2. Total transmission power versus expected SNRs of UEs for $O_{\max }=5$, $C_{\max }=5, M=6$

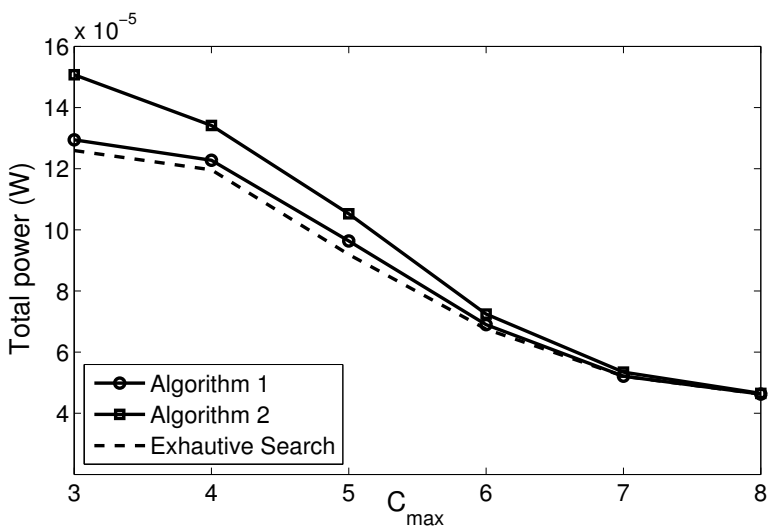

Fig. 3. Total transmission power versus $C_{\max }$ with $O_{\max }=5, \bar{\gamma}_{u}=30 \mathrm{~dB}$ $M=6$.

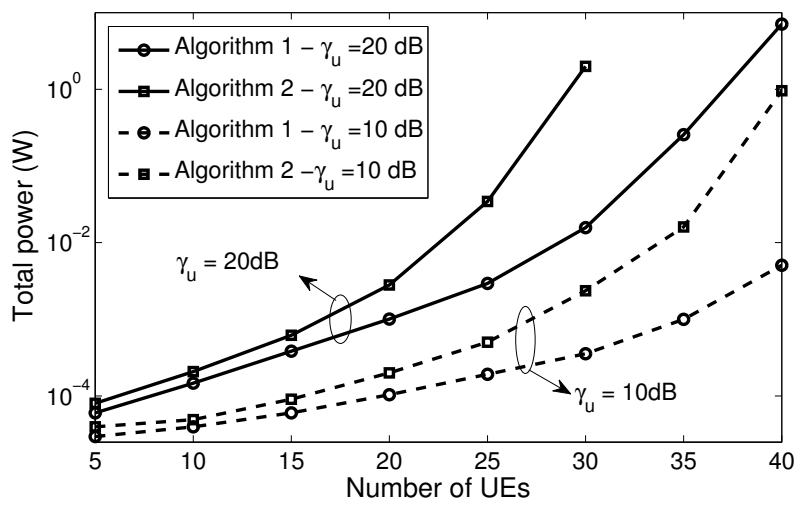

Fig. 4. Total transmission power versus the number of UEs in the large network where $K=16, O_{\max }=10, C_{\max }=5$.

Algorithm 1 results in just slightly higher power than that due to the optimal solution. Moreover, for low values of target SINR, the total power transmission of all schemes are almost the same.

In Fig. 3, we present the total transmission power of all RRHs versus the largest capacity of link from RRH to BBU $C_{\max }$ for different schemes. This figure confirms that
Algorithm 1 and Algorithm 2 perform well when the capacity of the fronthaul link is high. In addition, Algorithm 1 outperforms to Algorithm 2 and achieves the total transmission power close to that due to the optimum.

Finally, Fig. 4 illustrates the total transmission power of our proposed algorithms versus the number of UEs in a larger network where 16 RRHs are deployed as a square grid; the distance between two nearest RRHs is $50 \mathrm{~m}$; and the number of BBUs is 8 . In order to obtain each result, additional 5 UEs are randomly located inside a $150 \mathrm{~m} \times 150 \mathrm{~m}$ square of the RRH grid while the locations of the previously located ones are fixed. In this scenario, the exhaustive-search results cannot be obtained due to very large numbers of computations. As can be observed, the required transmission powers due to both lowcomplexity algorithms become higher with a larger number of UEs as expected. However, Algorithm 1 results in much lower transmission power compared to that due to Algorithm 2 as the number of UEs is large.

\section{CONCLUSION}

We have presented both optimal and low-complexity algorithms to minimize the total transmission power in CRAN subject to constraints on processing power at BBUs, the fronthaul capacity, and required QoS of users. Numerical results have illustrated the efficacy of our proposed lowcomplexity algorithms and the impacts of different parameters on the network performance.

\section{REFERENCES}

[1] M. Sawahashi, Y. Kishiyama, A. Morimoto, D. Nishikawa, and M. Tanno, "Coordinated multipoint transmission/reception techniques for LTE-advanced [Coordinated and distributed MIMO]," IEEE Wireless Commun., vol. 17, no. 3, pp. 26-34, Jun. 2010.

[2] R. Irmer, H. Droste, P. Marsch, M. Grieger, G. Fettweis, S. Brueck, H.P. Mayer, L. Thiele, and V. Jungnickel, "Coordinated multipoint Concepts, performance, and field trial results," IEEE Commun. Mag., vol. 49, no. 2, pp. 102-111, Feb. 2011.

[3] P. Marsch and G. Fettweis, "Uplink CoMP under a constrained backhaul and imperfect channel knowledge," IEEE Trans. Wireless Commun., vol. 10, no. 6, pp. 1730-Ü1742, Jun. 2011.

[4] "C-RAN: The road towards green ran," White Paper, China Mobile, 2011.

[5] "Suggestion on potential solution to C-Ran," NGMN alliance, Jan. 2013.

[6] N. Saquib, E. Hossain, Long B. Le, and D. I. Kim, "Interference management in OFDMA femtocell networks: Issues and approaches," IEEE Wireless Commun., vol. 19, no. 3, pp. 86-95, Jun. 2012.

[7] Vu N. Ha and Long B. Le, "Distributed base station association and power control for heterogeneous cellular networks," IEEE Trans. Veh. Technol., to appear.

[8] S.-H. Park, O. Simeone, O. Sahin and S. Shamai (Shitz), "Robust and efficient distributed compression for cloud radio access networks," IEEE Trans. Veh. Technol., vol. 62, no. 2, pp. 692-703, Feb. 2013.

[9] S.-H. Park, O. Simeone, O. Sahin and S. Shamai (Shitz), "Robust layered transmission and compression for distributed uplink reception in cloud radio access networks," IEEE Trans. Veh. Technol., to appear.

[10] C. Liu, K. Sundaresan, M. Jiang, S. Rangarajan, and G.-K. Chang, "The case for re-configurable backhaul in cloud-RAN based small cell networks," in Proc. IEEE INFOCOM, 2013.

[11] M. Grant, S. Boyd, and Y. Ye, "CVX: Matlab software for disciplined convex programming," 2009. Online: http://www.stanford.edu/ boyd/cvX.

[12] Z. Luo, W. Ma, A. M. So, Y. Ye, and S. Zhang, "Semidefinite relaxation of quadratic optimization problems," IEEE Sig. Proc. Mag., vol. 27, no. 3, pp. 20-34, May 2010.

[13] L. A. Wolsey, Integer Programming, John Wiley \& Sons, 1998.

[14] D., Jungnickel, Chapter Weighted matchings in Graphs, Networks and Algorithms, Springer Berlin Heidelberg, vol. 5, pp. 419-456, 2008. 